\title{
Communication Institutionnelle Et Recrutement Des Étudiants À L'université Félix Houphouët-Boigny De 2004 À 2009
}

\section{N'zué Yao Sylvain}

Département des Sciences et Techniques de la Communication, Doctorant au

Centre d'Études et de Recherche en Communication (CERCOM),

UFR Information communication et Arts (UFRICA), Université Félix Houphouët-Boigny, Abidjan, Côte d’Ivoire

doi: 10.19044/esj.2016.v12n13p445 URL:http://dx.doi.org/10.19044/esj.2016.v12n13p445

\begin{abstract}
The Ivorian idiosyncrasy on the market of the French speaking universities in the south of Sahara is characterized by the overabundance of students. That is why we try to understand how corporate advertising contributes to the popularization of the practices of the student "clientele" recruitment process at the university Félix Houphouet-Boigny of Cocody.

This work is essentially qualitative with the use of the direct observation technics and the semi-directive interviews with 20 university staffs of student recruitment process. It emerges from it that, on the one hand, the corporate advertising strategies during that operation of recruitment appear in three relation systems: the connections banking on the informal communication through affinities (kinship, friendship), the relations of transactions based on the communication of closeness and the relation of control centered on marketing approach.

On the other hand, these strategies establish partly real irregularity sources due to the pouring of thousands (parallel recruitment) of the high school diploma new holders in that public university without taking into account their secondary school performances and the accommodation capacity. This results in the overabundance of students and the worsening of the education quality.
\end{abstract}

Keywords: University, corporate advertising, external communication, public relations, students

\section{Résumé}

L’idiosyncrasie ivoirienne dans le marché des universités publiques d’expression française au sud du Sahara est caractérisée par les effectifs 
pléthoriques. C’est pourquoi nous essayons de comprendre comment la communication institutionnelle contribue à la vulgarisation du processus des pratiques de recrutement de la clientèle «étudiante » de l'Université Félix Houphouët-Boigny de Cocody.

Ce travail est essentiellement fondé sur l'approche qualitative traduite par l'observation directe et des entretiens semi-directifs auprès de 20 acteurs du processus de recrutement des étudiants. Il en ressort d'une part que les stratégies de communication institutionnelle au cours cette opération de recrutement se déclinent en trois systèmes relationnels : la communication informelle d'affinités (parenté, amitié), les relations de transactions basées sur la communication de proximité et les relations de contrôle axées sur la communication (marketing). D'autre part, ces stratégies constituent en partie de véritables sources d'irrégularités avec le déversement (recrutements parallèles) de milliers de nouveaux bacheliers dans les universités publiques sans tenir compte de leurs performances scolaires et de la capacité d'accueil des infrastructures : source de massification et de dégradation de la qualité de l'enseignement.

Mots-clés : Université, communication institutionnelle, communication externe, relations, Publiques, clientèle «étudiante»

\section{Introduction}

$\mathrm{Au}$ lendemain de la décolonisation et du mouvement de la montée des nationalismes, les intellectuels africains décident de guider la destinée de leur continent en s'appropriant le legs colonial afin d'africaniser le système éducatif dans le but de mieux orienter les politiques nationales. Dès cette prise de conscience, des universités vont se créer dont la première à Dakar pour l'Afrique Occidentale Française au Sénégal en 1957. Dès cet instant, l'ambiance générale des mutations dans les sociétés africaines va aboutir à un mouvement d'ensemble durant la deuxième décennie après les indépendances de 1960.

A ce titre, nous avons la création «en 1962 de l'université du Cameroun ,la Côte d'Ivoire, le Rwanda, le Burundi en 1964, la République Centrafricaine en 1969, le Togo, le Bénin, le Gabon en 1970, le Congo Brazza en 1971, le Niger en 1973, le Burkina Faso en 1974, la Mauritanie en 1981 et la Guinée Conakry en 1984 », note ZINSOU (2009:18). Ces universités vont faire la fierté de ces pays car elles sont une étape de l'affirmation de leur maturité.

Dans les années 1970, au sortir des indépendances, les universités africaines commencent à rencontrer leurs premières crises importantes (moyens financiers insuffisants, difficultés de redéfinition de leur politique) ${ }^{1}$. On assiste ainsi à des universités livrées à elles-mêmes s'efforçant de faire 
face aux exigences et défis du développement. Ces difficultés vont s'aggraver dans les années 1980 avec la rupture des grandes équilibres macroéconomiques (les programmes d'ajustement structurel) qui vont entrainer non seulement la détérioration des conditions d'étude et d'encadrement mais aussi une inadaptation accrue de l'enseignement aux réalités socio-économiques. Les universités entrent dès lors dans un cycle infernal de crises qui remet en cause l'ensemble de leurs dispositifs fonctionnels.

En Côte d'Ivoire, le fonctionnement de l’Université Félix HouphouëtBoigny sera mis à mal avec acuité avec la naissance de la Fédération Estudiantine et Scolaire en 1990 et par la crise poste électorale de 2002 dans lesquelles s'étaient enlisés les enseignants ainsi que leurs étudiants de façon cloisonnée. Ces deux acteurs n’ont jamais eu d'initiatives solidaires. Cette situation finira par aboutir à sa fermeture aux titres des années académiques 2010-2011 et 2011-2012. La qualité des structures d'enseignement ainsi que les conditions de vie et de travail des enseignants et des étudiants satisfaisantes jusqu'aux années 1980 se sont fortement dégradées.

Malgré ces conditions difficiles, l’Université Félix Houphouët-Boigny tente de développer des capacités de recherche de solutions afin d'optimiser son fonctionnement (séminaires, formations du personnel administratif, conférences, salon des universités). C'est dans cette dynamique que nous nous intéressons aux pratiques de recrutement des effectifs étudiants de cette organisation universitaire de 2004 à 2009. Quand on observe cette période (de l'étude) en termes de recrutement, rien ne présage la pratique d'opérations objectives d'orientation de bacheliers du fait de la crise qui secouait le pays. Cette situation sera couronnée par le transfert irrégulier de milliers d'étudiants orientés dans les autres universités publiques et établissements privés vers l’Université Félix Houphouët-Boigny : sources de dysfonctionnement. C'est dans cet esprit que nous nous posons les questions suivantes :

- Quel est l'apport de la communication institutionnelle dans les stratégies de recrutement des effectifs étudiants de l’Université Félix Houphouët-Boigny?

- Quelles sont les forces et les faiblesses des opérations de recrutements des effectifs étudiants de l'institution académique de Cocody?

Nourrissant l'ambition de contribuer à l'amélioration des pratiques de recrutement des effectifs étudiants de cette université, l'étude vise les objectifs suivants : 
- Déterminer l'apport de la communication institutionnelle dans les opérations de recrutement des effectifs étudiants de l’Université Félix Houphouët-Boigny ;

- Déterminer les forces et les faiblesses des opérations de recrutements des effectifs étudiants de l'institution universitaire de Cocody.

L'hypothèse sur laquelle se fonde cette étude avance que les nouveaux bacheliers sont recrutés à l'université Félix Houphouët-Boigny de Cocody durant cette période de crise sans tenir compte de leurs aptitudes (performance scolaire) et de la capacité d'accueil des infrastructures. L'on a l'impression que les autorités universitaires se débarrassent d'eux en les affectant dans des filières qui ne correspondent pas à leur profil.

C'est pourquoi l'objet de cet article tente de mettre en exergue dans une analyse essentiellement qualitative, l'importance de la communication institutionnelle dans les opérations de recrutement de la clientèle étudiante dans un établissement supérieur ivoirien représentatif, à savoir l'université Félix Houphouët-Boigny dans un contexte de ni guerre ni paix.

\section{I- $\quad$ spécification de la problématique}

\section{1-1 justification du choix du sujet}

Le choix du thème " communication institutionnelle et recrutement des effectifs étudiants de l’Université Félix Houphouët-Boigny de 2004 à 2009 » se justifie par le fait que depuis les années académiques 2004-2005 et 2005-2006, nous observons un véritable accroissement des effectifs étudiants dans nos universités publiques. La principale caractéristique de cette évolution affiche une volonté politique de remplissage des infrastructures en ne prenant pas en compte leur capacité d'accueil. Les fortunes sont donc de ce fait diverses car certains bacheliers, pour les plus chanceux seront recrutés dans les institutions universitaires publiques de leur choix, d'autres dans les grandes écoles privées et ceux dont les parents sont aisés se retrouvent dans les universités privées.

Conçue à l'origine pour 6000 étudiants, celle de Cocody fait face aujourd'hui au phénomène de la massification (progression continuelle des effectifs d'étudiants) comme l'indique ci après le tableau d'évolution du nombre de bachelier de 2004 à 2009.

Tableau 1 : effectif des nouveaux bacheliers de 2002 à 2009

\begin{tabular}{cc}
\hline Année & Nombre de Bacheliers \\
\hline 2004 & 34000 \\
2005 & 32418 \\
2006 & 46526 \\
2007 & 32266 \\
2008 & 38088 \\
2009 & 30000 \\
\hline
\end{tabular}

Source : Ministère de l'enseignement supérieur et de la recherche Scientifique, 2009 
Avec l'avènement de l'enseignement supérieur de masse, la physionomie de la population étudiante ivoirienne s'est considérablement modifiée. Il en résulte une croissance des effectifs entre 2004 et 2009 et une diversification des origines sociales. On remarque également une insuffisance au niveau des politiques d'orientation. Tous les étudiants n’ont pas accès aux filières correspondant à leur profil. La plupart est orientée dans des UFR de grandes capacités d’accueil ou dans des établissements privés d'enseignement supérieur.

L'annuaire statistique 2011-2012 du ministère de l'enseignement supérieur et de la recherche scientifique ivoirien illustrant ce que nous disons, nous conduit aux statistiques suivantes :

- $46 \%$ des étudiants sont inscrits en 2009 dans les universités publiques ;

- $16 \%$ dans les grandes écoles publiques en 2009 ;

- Les données chiffrées concernant les établissements privés d'enseignement et les institutions hors tutelle du ministère de l'enseignement supérieur et de la recherche scientifique ne sont pas disponibles.

Cette situation traduit clairement la volonté des autorités ivoiriennes d'affecter la plupart des bacheliers ivoiriens éprouvés d'ailleurs par plusieurs années de crise dans des structures sous tutelle sans se soucier des capacités d'accueil des institutions universitaires qui croulent elles-mêmes déjà sous le poids du boom d’effectif. L’étudiant ivoirien qui faisait la fierté de tous dans un passé récent est aujourd'hui perçu comme un paria. C’est pourquoi ce travail tente de comprendre comment les pratiques de recrutement des effectifs étudiants au sein de l’Université Félix HouphouëtBoigny sont mises en place.

\section{II- problème de recherche}

Sous le régime de la loi cadre Gaston Defferre du 23 juin 1956 qui accorda l'autonomie aux anciens territoires français de l'Afrique occidentale, le centre d’enseignement supérieur d'Abidjan créé le 31 juillet 1959 devint l'embryon de l'Université d'Abidjan. Les enseignements qui y étaient dispensés se faisaient sous la tutelle de l'Université de Dakar. Il comprenait trois écoles : les écoles de droit, de sciences et de lettres.

Les débuts satisfaisants de ce centre d'enseignement supérieur ont encouragé sa transformation en université adaptée aux réalités de notre pays comme l'indiquait en substance le père fondateur de la Côte d'Ivoire moderne Félix Houphouët Boigny en 1963 dans son discours sur les universités: "notre université devra faire intimement partie du corps social dont elle sera la plus haute expression culturelle. Elle devra exercer les fonctions de gardienne et de défenseur du patrimoine de la communauté 
africaine, tant sur les plans philosophique et linguistique que dans les domaines des arts, de la littérature et de la musique ». C'est dans ce sens que Zinsou (2009: 16) soutient qu' «une université n'est jamais créée en dehors de la société ». L’université doit être la cellule incubatrice des politiques de développement pour faire face aux exigences de la mondialisation. Telle fut la mission remplie par celle de Cocody à travers la formation d'élites (ivoiriennes et africaines) conformément à la conclusion de la conférence de Tananarive en 1962 qui recommande aux pays africains d'accorder une priorité à la formation des cadres scientifiques.

C'est donc officiellement le 9 juillet 1964 que le gouvernement ivoirien créa l'Université d’Abidjan qui tournait à plein régime dans un climat de paix grâce aux performances économiques remarquables l'État.

L’université, à cette époque, était régie par une discipline et le respect de la hiérarchie. Les étudiants étaient effectivement affectés dans les filières de leur choix conforment à leur profil. Les cours se déroulaient sans véritables perturbations et l'étudiant, au sein de la société, bénéficiait d'une image positive.

Cette atmosphère propice aux études et favorable à l’épanouissement des étudiants sera progressivement détériorée à cause de la baisse des cours des matières premières en 1980. Dès cet instant, les états africains connaissent des difficultés économiques, politiques et sociales.

Sous l'impulsion des organismes internationaux, les programmes d'ajustement structurels (PAS) sont mis en place. C'est dans cette dynamique que Goin $\mathrm{Bi}$ (2011:136) affirme que: «l'enseignement supérieur est marginalisé au profit de la formation de base » car les financements seront désormais orientés vers l'éducation de base.

En 1990, les difficultés vont s’accentuer avec la conférence de la Baule prononcée le 20 Juin 1990 par le Président Français François Mitterrand (1916-1996) qui invitait les pays africains à accélérer leur démocratisation en vue d'une pérennisation des échanges avec le nord. Elle va de ce fait donner le ton du multipartisme avec son lot de revendications d'ordre corporatiste en Côte d'Ivoire. L'université se trouvant au centre de toute sociabilité, celle de Cocody n’échappera pas à ce bouleversement, notamment avec la naissance de la Fédération Estudiantine et Scolaire de Côte d'Ivoire en avril 1990 (FESCI) en tant que mouvement principal de la défense des droits et intérêts des étudiants. Dès cet instant, l’Université Félix Houphouët-Boigny va connaître une instabilité constante parce que la FESCI se signale par de multiples grèves qui sont la plupart du temps suivies de casses. Face à la fermeté du pouvoir d'alors (PDCI-RDA), ce mouvement s'est détourné de son arme de combat initiale (grève et négociation) pour s'inscrire dans la violence comme ultime arme de combat de revendication. Ce mouvement estudiantin va donc se positionner comme un instrument politique qui va 
entretenir la fronde durant plusieurs années sur les campus universitaires ivoiriens en organisant la vie de ces temples du savoir selon ses lois faisant fi des libertés académiques.

A cela , il faut ajouter d'une part que conçue initialement pour 6000 places, l'institution universitaire de Cocody comptait en 2009 environ $65892^{3}$ étudiants régulièrement inscrits, source de problèmes de tous genres: effectifs pléthoriques, classes et amphithéâtres insuffisants, équipements didactiques insuffisants ou (inadéquats, voire inexistants), ratio professeurs / étudiants défavorable, structures d'accueils et hébergement des étudiants insuffisants ou peu opérationnelles, etc. et d'autre part, la crise poste électorale de 2002 qui a fait tourner véritablement la haute école de Cocody au ralenti. C’est pourquoi BLÉ Raoul G. (2009:3) affirme que : « la crise des universités publiques dans la crise ivoirienne n'est en réalité que les conséquences de l'incapacité des dirigeants depuis 1990 à maitriser une telle organisation ».

Numériquement faible, les $1722^{4}$ enseignants ne sont plus à mesure d'offrir une formation de qualité aux étudiants. L'on assiste à la démission des principaux acteurs (gouvernants et autorités académiques) du système éducatif ivoirien. Conscients de la lourde tâche qui est la leur, les enseignants deviennent les maîtres à tout faire de cette sphère académique pour essayer de sauver les nombreuses années académiques juxtaposées. C'est dans ce sens que BLÉ Raoul G (2009:2) renchérit pour dire que : « il est fréquent de voir au quotidien, sur le campus, des enseignants et leurs étudiants se promener, à la recherche d'une salle de cours ».

De ce point de vue, il n'est pas exagéré de dire que l'Université Félix Houphouët-Boigny est au terme de son développement initial. Elle est à la fin de ses missions originelles (production et transmission du savoir). Dans ce contexte, l'orientation académique qui vise à réguler le flux d'entrée dans l'enseignement supérieur tout en affectant les étudiants dans les filières qui correspondent à leur profil présente des insuffisances du fait de la massification dans laquelle est plongée l'institution universitaire de Cocody. La qualité des services d’orientation a donc une incidence certaine sur la performance des étudiants. C'est dans cette perspective que Makosso (2009:88) avance qu' : "en côte d'ivoire, les services d'orientation sont très peu sollicités : 83 pour cent des étudiants choisissent eux-mêmes leur filières. Ils méconnaissent de ce fait les débouchés professionnels associés aux différentes filières et les possibilités de trouver un emploi en terme de leur formation ».

À en croire Makosso, les choix de la plupart des étudiants ivoiriens s'opèrent par mimétisme empêchant ceux-ci d'être performants dans des filières qui ne correspondent pas à leur profil. Ce qui les amène à vouloir changer de filières au fil des années académiques. 
Ce tableau diagnostic qui révèle la non-maitrise du flux migratoire et l'orientation de la clientèle étudiant nous amène à poser la question suivante : comment la communication institutionnelle se met au service des stratégies de vulgarisation des opérations de recrutement de la clientèle étudiante à l’Université Félix Houphouët-Boigny?

\section{III- Inscription Théorique et Méthodologique \\ 3-1 Approches théoriques \\ 3-1-1 Définition des concepts}

Dans un travail scientifique, la définition des mots-clés et des concepts est importante dans la mesure où ils permettent de mettre les lecteurs et le producteur du discours au même niveau de compréhension.

\subsubsection{Communication institutionnelle / Communication Corporate}

La communication corporate est apparue dans les années 1920 aux Etats-Unis et a subi une évolution actuellement avec la récession qui incite les entreprises à investir plutôt dans des actions de court terme où la rentabilité se mesure plus instantanément. C'est pourquoi Thierry Libaert (2009:76) l'appréhende comme le «domaine de la communication externe qui traite de l'entreprise en tant qu'institution, d'où son nom de communication institutionnelle ». Elle se fonde sur des thèmes principaux portant sur la puissance, le leadership, les activités, la capacité d'innovation, le sens du service et les relations que l'entreprise souhaite entretenir avec ses clients.

Pour N. Gadal (1992:74) « la communication corporate est un ensemble de fiches signalétiques : politiques, stratégies, organisation, fonctionnement, ambitions, à partir desquelles l'entreprise se fait connaître à tous ses partenaires (internes, externes) sous deux aspects fondamentaux à savoir de personne morale ou de personne créatrice de bien ou de services".

Pour lui, la communication corporate contribue à asseoir l'image de marque d'une organisation, à renforcer sa crédibilité et la confiance de son environnement. C'est pourquoi Bernard Lamizet et Hamed silem (1997:131) soutiennent que «la communication corporate est un discours des organisations sur elles-mêmes ».

Pour une entreprise, selon ces auteurs, il s'agit de faire connaître l'organisation, d'identifier ses activités et de donner une bonne image d'ellemême à ses publics tant en externe (élus, clients potentiels, fournisseurs, banques) qu'en interne (salariés, actionnaires, représentants syndicaux), d'exprimer de la sorte, sa légitimité économique et sociale.

L'élaboration de politiques de communication en faveur de la promotion des produits (diplômes) et mise à la disposition du grand public des plates formes afin d'informer en temps réel le grand public et particulièrement les 
nouveaux bacheliers et parents d’élèves demeure un rituel à l'université de Cocody.

\subsubsection{Relations publiques}

Ensemble d'actions servies par des techniques et des procédées divers dont le but est de faire connaître et de faire valoir l'image de marque d'une entreprise, d'une institution, ou d'une personne, les relations publiques (RP) seront définies dans un contexte beaucoup plus global en nous appuyant sur Frédérique De Meulemeerter (1991:13) et N.Gadal (1992 :71) pour illustrer notre propos.

Pour le premier, les relations publiques sont : « un ensemble d'actions de projection de l'image institutionnelle de l'entreprise pour faire savoir ce qu'elle est. Ce qu'elle fait. Et comment elle s'efforce de répondre aux attentes de ses partenaires, afin de générer une confiance réciproque ».

De ce point de vue, l'action des relations publiques à l'Université Félix Houphouët -Boigny doit être alors permanente, planifiée à long terme, coordonnée avec celle de tous les départements de l'institution, au service de sa réputation.

Pour le second : les relations publiques permettent de définir et de mettre en œuvre, de manière continue ou ponctuelle, tout ou partie d'une politique de communication, d'information, au service d'une entreprise, d'une administration, d'une collectivité, ou de toute autre entité - qu'elle soit personne morale ou physique - en direction de ses publics et interlocuteurs. Qu'ils soient internes ou externes ».

C'est d'ailleurs pour cette raison qu' Alex Mucchielli (2001: 66) affirme que «les relations publiques sont l'ensemble des actions de communication par lesquelles une entreprise cherche à susciter un courant d'estime et de confiance en sa faveur en informant le grand public de ses activités et réalisations (journées portes ouvertes, parrainage d'activités culturelles, scolaires, humanitaires ou sportives ». Pour Alex Mucchielli, les relations publiques visent à positionner le client par rapport à l'entreprise dans une relation favorable à celle- ci.

En ce qui nous concerne, les relations publiques constituent une stratégie publicitaire permettant à l'organisation universitaire de Cocody de se faire comprendre et écouter par ses divers publics tant internes qu'externes (personnel administratif et technique, enseignants, étudiants, nouveaux bacheliers et parents d’élèves).

\subsubsection{La communication externe}

Selon Bernard Lamizet et Hamed Silem (1997:130), la communication externe est « un ensemble d'actions de communication institutionnelles et commerciales entreprises par une organisation pour améliorer sa notoriété 
à l'extérieur auprès de ses différents publics : clients potentiels et effectifs, fournisseurs, financiers, actionnaires potentiels et effectifs, pouvoirs publics, citoyens, hommes politiques syndicats ».

Dans ce sens, il s'agit donc du champ de la communication d'entreprise qui milite en faveur de la valorisation du capital image de l'entreprise et du capital image du ou des produits que l'entreprise met sur le marché.

C'est pourquoi nous nous appuyons sur Alex Mucchielli (2001:67) pour dire que la communication externe comprend : "toutes les communications destinées à valoriser aux yeux de différentes cibles l'entreprise elle-même : son image ou ses produits ».

C’est cet aspect qu'Alain Milon et Michèle Jouve (1996 :7) soulignent d'ailleurs en ces termes "penser différemment les modes et lieux de fonctionnement de la communication d'entreprise. C'est permettre à l'entreprise de s'intégrer dans des nœuds de réseaux qui lui permettent d'acquérir plus de compétences afin d'externaliser ses tâches et d'augmenter non pas sa plus value ajoutée, mais ses chaînes de valeur ajoutée».

À la lumière de nos lectures, beaucoup d'entreprises (entreprises de type moderne) consacrent une grande partie de leur énergie à communiquer avec l'extérieur. C'est un objectif commercial et institutionnel primordial mais, il doit y avoir une synergie entre communication externe et la communication qui règne au sein de l'entreprise car une bonne communication interne rejaillit favorablement sur celle tournée vers l’extérieur.

\subsubsection{Revue de la littérature}

Pour mener à bien notre étude, il convient de faire la revue de littérature de plusieurs œuvres, documents et réflexions pour situer notre sujet par rapport aux recherches antérieures. Dans ce sens, il est important de relever quelques - uns de ces écrits qui s’inscrivent dans le champ de notre étude.

\subsubsection{Ecrits sur les universités au sud du Sahara}

Notre propos s'appuie sur la publication de René AIGRAIN (1949) « Histoire des Universités » qui livre la traçabilité historique de l'évolution de l'université de la période médiévale à la période des universités hors de l'Europe. Cet ouvrage est un guide théorique qui permet de comprendre l'essence de l'université en tant qu'organisation porteuse de solutions aux enjeux de développement. C'est pourquoi en 1979, Diarrassouba Valy Charles (recteur de l'université de Cocody de 1974 à 1983), dans son ouvrage «l'Université ivoirienne et le Développement de la Nation », vulgarise l'organisation et le fonctionnement de l'institution universitaire de Cocody. A travers cinq chapitres, il expose les enjeux culturel, organisationnel, économique et académique de cette haute école. Son 
approche est une démarche qui peut servir de référence dans le fonctionnement de l’Université Félix Houphouët-Boigny.

Cependant en 1972, Jean Fourastié, dans « Faillite de l’Université », soutient que «l'université à l'origine, institution porteuse d'espoir en termes de formation de l'élite se trouve confrontée à de nombreux dysfonctionnements qui doivent être pansés pour permettre à ces institutions de remplir leur objectif de politique de développement ». Dans le même ordre d’idées renchérit le Camerounais Paul John Marc Tedga (1988) dans son œuvre «Enseignement Supérieur en Afrique noire francophone, la catastrophe ? », en exposant dans huit chapitres les raisons de la faillite des universités en Afrique noire francophone.

Selon lui, l'héritage transmis par les universités marraines aux institutions universitaires d'expression francophone au sud du Sahara souffre de plusieurs crises d'ordre fonctionnel et organisationnel. De ce fait, les missions traditionnelles assignées aux universités africaines qui se résumaient pour l'essentiel aux notions de développement social et économique du continent africain n’ont pas été remplies car la majorité des pays s'enlisaient au fil des années dans des conflits interminables peu favorables pour la formation. Zinsou Michel (2009) s’inscrit dans le même ordre d'idées pour dire que les organisations universitaires notamment celle de Cocody est confrontée à de nombreuses difficultés d'ordre fonctionnel qu'elle se doit de solutionner pour qu'elle puisse jouer valablement le rôle qui est le sien.

À la lumière de ce qui précède, nous retenons que l'université Félix Houphouët-Boigny croule sous le poids de moult dysfonctionnements suscités par les crises économiques et politiques. C’est pourquoi nous couronnons notre lecture par Essane Séraphin (2001) dont l’ouvrage « une Sociologie de l'Université en Afrique» est une invitation aux autorités universitaires (communauté scientifique) et étatiques à restaurer et réhabiliter les institutions universitaires pour qu'elles deviennent (une priorité au sommet de l'état) de véritables espaces de formation de l'élite pour le développement de toute nation en Afrique.

\subsubsection{2 revalorisations de l'image institutionnelle des universités au sud du Sahara}

La question relative à la mise en valeur de l'image institutionnelle et de la problématique de communication institutionnelle dans les universités africaines en général et spécialement de l’université Félix Houphouët-Boigny a fait l'objet de plusieurs écrits.

Déjà, en 1964, l’Unesco dans : « l’avenir de l'enseignement supérieur en Afrique » jetait les bases d'une planification pour un enseignement supérieur de qualité en Afrique. C'est dans cette même dynamique qu’en 
2006, elle élabore avec l'organisation de coopération et de développement économique (OCDE) les lignes directrices destinées à garantir la qualité de l'enseignement supérieur transfrontalier. Dans un contexte de mondialisation, ces lignes directrices ont été développées en réponse à la commercialisation croissante de l'enseignement supérieur. Elles s'adressent tant aux pays développés qu'aux pays en développement. Leurs objectifs dans l'ensemble étaient destinés à proposer des outils susceptibles d'aider les pays à évaluer la qualité et la pertinence de l'enseignement supérieur transfrontalier et de protéger les étudiants et autres parties prenantes contre les services de qualité médiocre. Les lignes directrices s'adressent aux principales parties prenantes de l'enseignement supérieur : gouvernements, établissements et prestataires d'enseignement supérieur, associations étudiantes, organismes d'assurance qualité et d'accréditation, organismes de reconnaissance des qualifications académiques, organismes professionnels.

Cependant, face à la triple contrainte d'une demande sociale d'enseignement supérieur forte, de marges budgétaires réduites et d'un marché de l'emploi peu porteur, les choix de politiques s'avèrent limités pour la plupart des pays selon Pierre-Antoine Gioan (2006) dans: " enseignement supérieur dans les pays francophones d'Afrique : tendances et défis ", qui propose trois principes directeurs en termes de politiques de réhabilitation de l'enseignement supérieur africain à moyen terme :

$\checkmark$ Renforcer l'utilité sociale et économique de l'enseignement supérieur et de la recherche en adaptant au maximum l'offre de formation et de recherche aux besoins réels des pays ;

$\checkmark$ Maitriser l'évolution des effectifs étudiants dans des proportions compatibles avec les possibilités budgétaires ;

$\checkmark$ Optimiser l'utilisation des moyens disponibles en impliquant au mieux le secteur privé et en orientant le plus possible les ressources disponibles vers les dépenses pédagogiques et de recherche.

C'est fort des observations de Pierre-Antoine que Varghese (2004) fait remarquer par le canal de son article: «incitations et changements institutionnels dans l'enseignement supérieur » que les systèmes éducatifs du monde entier continuent de dépendre en grande mesure des ressources publiques de financement. Les dépenses consacrées à l'enseignement supérieur sont couvertes à près de $80 \%$ par les fonds publics dans les pays de l'OCDE et cette proportion est plus forte encore dans les pays en développement. Nombre de pays s'efforcent actuellement de réduire cette dépendance vis-à-vis des crédits publics et s'orienter vers des réformes plus fondées sur le jeu du marché. Pour l'auteur, Ils doivent, pour ce faire, aménager le cadre des politiques macroéconomiques afin d'entrainer des changements au niveau des établissements. Les changements institutionnels peuvent être induits au moyen de mandats ou de récompenses. Les mandats 
exigent des établissements qu'ils adoptent une forme particulière de comportement institutionnel. En revanche, les récompenses offrent des motivations et des incitations au changement. Il est donc question dans cet article de la possibilité de faire changer les établissements d'enseignement supérieur grâce aux incitations. Dans le même ordre d'idées renchérissent Michael Harloe et Beth Perry (2005) avec: « repenser l'université sans la vider de son sens: engagements externes et transformations internes de l'université dans l'économie du savoir » pour dire que le développement de l'économie du savoir place les universités au cœur du processus de mutation économique en rapportant avec leurs fonctions d’enseignement, de recherche et d'ouverture. Et que cet engagement contraint les universités à considérer la nécessité de transformations internes visant à se hisser à la hauteur de leurs nouveaux rôles, plus entrepreneuriaux. C'est d'ailleurs cet aspect des réformes qu'ont fait observer Séraphin Alava et Louise Langevin (2001) dans : « l'université, entre l'immobilisme et le renouveau ». Pour ces auteurs, l'équilibre à maintenir entre le passé, le présent et l'avenir, entre l’héritage culturel à transmettre et les mutations à prendre en compte, oblige l'université à se renouveler. Ils ajoutent que c'est par l'observation critique, par la réflexion sur ces propres composants, par le maintien d'une vitalité pédagogique rénovée que l'université pourra éviter la robotisation de l'apprenant, la déshumanisation de l'institution. Ces mutations qui la menacent remettent l'université face à ses missions fondamentales : créer des savoirs, former une pensée libre, initier à la citoyenneté. Elle parviendra à maintenir le cap en innovant pour son propre compte. Pour ceux-ci, c'est à ce prix que l'université pourra atteindre ses objectifs et bénéficier d'une autonomie qui lui permettra de faire face aux pressions internes et aux mutations externes. C'est pourquoi Hamidou Nacuzon Sall (2009) avec: « enjeux et perspectives d'une réforme institutionnelle: les universités africaines face au processus de Bologne » fait observer que les universités africaines doivent être particulièrement attentives au contexte mondial actuel fortement marqué par le courant néolibéral qui amène à la marchandisation de l'éducation d'une part et par la recherche de normes universellement comparables dont relèverait le processus de Bologne d'harmonisation des cursus et des diplômes en Europe d'autre part. Le juste équilibre semble difficile à trouver entre exigences en apparence peu conciliables avec le développement d'un système éducatif démocratique et de qualité, englobant les secteurs formel et non formel, dans des pays pauvres. L'une des missions essentielles de la recherche universitaire de qualité est justement de contribuer à construire des éléments de réponses à toutes ces questions. C'est donc à juste titre que François belle et Claude Echevin (1992) à travers: «l'émergence de la communication institutionnelle à l'université : enjeux et perspectives » révélaient 
l'importance de la communication institutionnelle dans le fonctionnement de tout établissement d'enseignement supérieur. Ils ajoutent que les universités mettent en œuvre des actions de communication institutionnelle tant pour mieux se faire connaître de leur environnement que pour faire évoluer leur culture interne.

En conclusion, la communication institutionnelle est une stratégie de séduction du grand public notamment la clientèle étudiante. Elle est une communication d'appel de fonds et de recrutement qui s'appuie sur les éléments de notoriété : réputation des enseignants, conditions de recrutement des étudiants et les travaux de recherche des universités.

\section{3-1-3 Inscription Paradigmatique}

L'une des exigences scientifiques pour assoir une théorie commande de parcourir d'abord celles qui existent.

\section{3-1-3-1 Cadre de référence théorique}

Notre positionnement théorique va se nourrir d'un mix théorique c'est-àdire l'utilisation harmonieuse de deux théories fondatrices des sciences de l'information et de la communication. Nous appuyons sur les approches théoriques, notamment le modèle mathématique de l'information de Claude Shannon et Warren Weaver et l'approche managériale qui nous paraissent fondamentales dans la compréhension du fonctionnement de l'Université Félix Houphouët-Boigny.

\subsection{La théorie mathématique de l'information de Claude Shannon (1916) et Warren Weaver (1896-1978)}

Dans une approche de la communication transmission, nous nous appuyons sur les travaux de Warren Weaver et de Claude Shannon pour comprendre le processus de communication et la direction de l'information (horizontale, verticale ou ascendante) pour en mesurer la portée des messages qui s'établissent entre l'administration de l'Université Félix Houphouët-Boigny et le grand public.

C'est pourquoi nous nous appuyons sur le système d'information de cette institution académique pour illustrer ce que nous disons. Nous appelons système d'information le dispositif par lequel la haute école de Cocody s'informe pour gérer son fonctionnement et son évolution. Ce système se nourrit de trois entités fondamentales : le personnel, l'organisation et les technologies de l'information et de la communication.

\section{$\checkmark$ Le personnel}

Font partie du système d'information les personnes qui sont à l'origine des informations, qui les font circuler, les traitent et les utilisent. On comprend donc que peu de membres de l'entreprise universitaire de Cocody 
sont exclus du système d’information. Ceci donne à penser que gérer le système d'information de l'institution académique de Cocody, c'est avant tout avoir affaire à des hommes. Cela suppose donc qu'on veuille et qu'on sache les écouter, les convaincre, les motiver, les affronter et négocier.

\section{$\checkmark$ L'organisation}

Par l’organisation, nous désignons notamment l’organisation du travail à l'université Félix Houphouët-Boigny. La façon dont le travail est reparti en tâches, les caractéristiques des tâches, les dispositifs de contrôle, les langages utilisés par les uns et les autres, la culture universitaire, les règles et les procédures de gestion. En somme, il s’agit de mettre en œuvre la dimension organisationnelle du système d'information.

\section{$\checkmark$ Les technologies}

Les technologies mises en œuvre dans le système d'information sont de plus en plus nombreuses et diverses touchant l'acquisition des informations, la communication, le stockage et l'exploitation des informations. Les technologies de l'information ne sont pas des outils. Ils sont choisis compte tenu des besoins et des caractéristiques des informations concernées. Au sein de l'organisation universitaire de Cocody, cohabitent des dispositifs d'information et de communication traditionnelle et nouvelle qui sont d'une hétérogénéité croissante: journaux universitaires internes, internet, GED (gestion électronique de document), logiciels de base de données, de microordinateurs et d’imprimantes, la communication électronique : outils de veille informationnelle. Ils contribuent à améliorer la communication et les flux informationnels, dont la maitrise est primordiale afin de maintenir un haut niveau d'innovation et de compétitivité.

En somme, le système d'information et de veille occupe ainsi une place importante dans le management de l'établissement supérieur de Cocody car ils fournissent d'une part des informations externes, relatives à l'environnement et d'autre part des informations internes, spécifiques à la haute école de Cocody.

\subsection{L'approche managériale}

Ici, il est nécessaire de souligner que la notion d'organisation fait référence à la pertinence du système et du mode de gestion ou management de l'Université Félix Houphouët-Boigny. C'est pourquoi nous nous appuyons sur le management participatif, pour observer le mode de gestion de cette institution. A cet effet, des icônes du management, dont Minzberg, Simon, Walter, de l'école des relations humaines, nous guideront à travers leurs réflexions.

Pour ces auteurs, chaque employé doit être informé de la vie de l'entreprise dans le but de mieux informer et répondre aux attentes des clients. 
À l’Université Félix Houphouët-Boigny, en termes de circulation et de gestion de l'information (système d'information), l'on peut avancer que le processus de communication prend généralement sa source dans la hiérarchie de l’institution (présidence, vice - présidences, secrétariat général).

Ces émetteurs transmettent les informations aux différents directeurs d'UFR, d'instituts, de centres, responsables syndicaux et étudiants (récepteurs) grâce aux canaux de transmission dont les plus utilisés sont :les notes de service, d'information, les circulaires et les journaux d'université pour informer au quotidien leurs proches collaborateurs ainsi que le grand public.

La transmission des messages part aussi de la base de l'organigramme (chefs de service, responsables syndicaux, enseignants, étudiants) qui deviennent à leur tour des émetteurs. Ceux-ci à travers la transmission de leurs différents messages font part de leurs préoccupations ou aspirations à la hiérarchie de l'institution.

$\mathrm{Au}$ total, la gestion du flux d'information à l'Université Félix Houphouët-Boigny se fait de manière horizontale et verticale (descendante et ascendante).

Toutefois, ce type de gestion participatif mérite d'être amélioré par le modèle de l'empowerment qui donne plus de responsabilité et de marge de manœuvre aux membres du personnel pour une université de performance (travail de cohésion et d'équipe).

De manière globale, ces actions de communication visent à établir un climat psychologique favorable entre l'université ses publics interne (personnel administratif et technique, enseignants, responsables syndicaux et étudiants) et externe :(clientèle étudiante, parents et partenaires). Elles s'inscrivent dans les stratégies relationnelles institutionnelles développées par la Direction de l'université vers son environnement axé sur la notion de culture d'entreprise qui met en exergue les règles et les procédures de gestion de l'entreprise universitaire de Cocody. C'est pourquoi les journaux d'université : Université et Les Cahiers du Personnel de l'université FélixHouphouët-Boigny de Cocody sont susceptibles de développer un sentiment d'appartenance (levier de performance) en faisant connaître aux membres de l'institution les activités qui s'y déroulent.

\subsection{Cadre méthodologique}

Cette étude a été réalisée à l'Université Félix-Houphouët-Boigny de Cocody sur une période deux mois: avril à mai 2014. La population concernée se compose des responsables de services impliqués dans la mise en place des opérations de recrutement à savoir: le Secrétariat Général, le Directeur de la Scolarité Centrale, les Directeurs centraux, le chef de Service de communication et les inspecteurs d'orientation. Ils sont au nombre de vingt personnes. 
Cette enquête essentiellement qualitative fondée sur des entretiens semidirectifs à travers des guides d'entretien se résume pour l'essentiel autour des préoccupations suivantes :

- Les outils de communication institutionnelle de l’Université FélixHouphouët-Boigny ;

- Les stratégies et outils de recrutement des effectifs étudiants ;

- Les forces et faiblesses de ces différentes politiques de recrutement ;

- Et enfin les apports de la communication dans le processus de recrutement de la clientèle étudiante.

La collecte des données s'est également appuyée sur la technique d'observation directe qui sur le terrain, nous a permis d'observer les pratiques communicationnelles durant ces deux mois à travers une grille d'observation.

\section{- Traitement des données}

Le traitement de ces données qualitatives s'est appuyé sur une analyse de contenu des différents entretiens. Il s'agissait pour nous de traiter les informations récoltées en fonction de nos différents objectifs spécifiques. À savoir :

- Déterminer l'apport de la communication institutionnelle dans les opérations de recrutement des effectifs étudiants de l'Université Félix Houphouët-Boigny;

- Déterminer les forces et les faiblesses des opérations de recrutements des effectifs étudiants de l'institution universitaire de Cocody ;

Le but poursuivi dans cette phase de l'analyse a été de dégager, des discours des répondants, des significations qui mettent en lumière les stratégies de recrutements. Les données recueillies ont fait l'objet d'un traitement manuel. La méthode de l'analyse de contenu a donc consisté à catégoriser et à organiser les informations exprimées de façon très variée. Elle nous a permis de dégager des catégories d'analyse sur la base des informations recueilles. Il s'agit précisément de l'analyse de discours et de l'analyse lexico-thématique des réponses.

\section{Résultats et Discussion}

4-1stratégies de communication institutionnelle et recrutement des effectifs étudiants de l’Université Félix Houphouët-Boigny

\section{- La période 2004-2009}

Bien que peu planifiée et avec de faibles ressources, la question du recrutement des effectifs étudiants de l’Université Félix Houphouët-Boigny de Cocody était effective au cours de cette période. C’est la présidence (viceprésident chargé des enseignements) et le Directeur de la scolarité qui en sont les premiers responsables. L'entrée des étudiants se fait par deux voies : 
les préinscriptions pour les nouveaux bacheliers et le recrutement par analyse de dossiers d'équivalence de diplômes (fidélisation) pour les étudiants déjà inscrits dans une quelconque filière.

\section{- Les préinscriptions}

Peuvent postuler, les étudiants qui possèdent un baccalauréat (toutes les séries confondues) de l’année en cours. La stratégie de recrutement se nourrit ici de deux phases bien distinctes :

- la phase relative au cadre réglementaire (constitution de dossiers) qui obéit aux critères d'entrée dans les différentes filières) ;

- La deuxième se résume essentiellement à l'analyse du profil des nouveaux bacheliers en fonction des moyennes d'orientation en vigueur et de la capacité d'accueil des différentes unités de formation et de recherche (UFR) et de l'âge du candidat qui est de 23 ans au maximum.

\section{-dossier d'équivalence de diplômes}

Cette opération est destinée aux étudiants désireux de poursuivre leurs études dans d'autres filières. Elle est connue sous l'appellation formation individuelle professionnelle (FIP) qui donne la possibilité aux travailleurs aussi bien qu'aux étudiants ordinaires de poursuivre leur formation dans (d'autres) filières de leur choix.

\section{-Stratégies de communication}

Tout au long de la période de 2004 à 2009, la communication de proximité était présente par des stratégies de transmission plus ou moins explicites d'informations documentaires et de communication directe et de relation de presse (conférences, points de presse et séances d'information) entre les autorités universitaires, les nouveaux bacheliers et parents d'élèves d'une part et d'autre part entre les inspecteurs d'orientation et les nouveaux bacheliers. Ces pratiques communicationnelles sont assurées par le Directeur de la scolarité de l'université. Ses principaux moyens sont la production de prospectus, une communication (séances d'information) transmise aux nouveaux bacheliers en quête d'information et l'affichage. Cette phase de contact (entretiens : explication des différentes phases de l’opération de recrutement et les différents débouchés des choix opérés par les nouveaux bacheliers) est réalisée par les inspecteurs d’orientations et le service accueil.

\section{La période poste-crise}

Cette deuxième grande phase se caractérise par la croissance accélérée des effectifs étudiants et l'intervention (assistance) de l'État dans les affaires universitaires. L’immixtion du pouvoir politique dans les affaires des universités en Côte d'Ivoire est observable par la nomination des administrateurs, interventions policières dans les règlements des conflits internes aux établissements et l'affaiblissement des instances «institutionnelles» de régulation de conflits (syndicats). L'autoritarisme d'état et la perte d'autonomie de la communauté 
universitaire sont les paradigmes les plus patents d’une problématique de la crise institutionnelle à l'université. Cet assujettissement qui se caractérise par un manque d'autonomie des universités publiques devient de plus en plus manifeste du fait du mouvement de désengagement économique dans un contexte d'explosion de la population étudiante. Il revient donc aux institutions universitaires de mobiliser des fonds pour leur survie.

Cette assistance consiste à accompagner les institutions universitaires publiques pour mieux négocier l'après-crise en termes de fonctionnement. Les universités publiques de Côte d’Ivoire s’occupent de façon minimale du recrutement des effectifs étudiants, car le plan de recrutement est élaboré par le Ministère de l'Enseignement Supérieur et de la Recherche Scientifique à travers sa Direction de l'Orientation et des Examens (dorex) pour résoudre la question du flux migratoire des étudiants.

\section{-Stratégies de communication}

Au cours de la deuxième période en plus des stratégies de communication de proximité (informations documentaires, communication directe et les relations de presse), deux nouvelles stratégies de communication institutionnelle à savoir la communication électronique (préinscription, réinscription et paiement des frais d'inscription en ligne) et la communication interne se sont ajoutées aux stratégies de communication institutionnelle de l’Université Félix Houphouët-Boigny. La communication interne se présente dans ce contexte comme un outil permettant à la direction de cette institution de mettre en œuvre des actions de communication « institutionnelle » en interne. La communication interne a pour objectif ici de susciter un sentiment d'appartenance afin d'y instaurer une culture universitaire favorable (communication d’affinité : parenté ou amitié).

- Tableau 2 : Résumé des stratégies de communication selon les périodes analysées

Période Stratégies de liens $\quad$ Stratégies de Transactions $\quad$ Stratégies de Contrôle

$\begin{array}{llll}2004-2009 & \begin{array}{l}\text { Influence } \\ \text { Informelle }\end{array} & \begin{array}{c}\text { Informations documentaires } \\ \text { Communication directe } \\ \text { Relation presse }\end{array} & \text { publicitaire }\end{array}$

\begin{tabular}{|c|c|c|c|}
\hline $2009 \ldots$ & $\begin{array}{l}\text { Influence } \\
\text { Informelle }\end{array}$ & $\begin{array}{l}\text { Informations documentaires } \\
\text { Communication directe } \\
\text { Relation de presse } \\
\text { Communication interne } \\
\text { Communication électronique }\end{array}$ & $\begin{array}{l}\text { Publicitaire } \\
\text { Image de marque } \\
\text { Promotionnelle }\end{array}$ \\
\hline
\end{tabular}

Les stratégies de communication institutionnelle de l'université de Cocody se déploient de manière non exclusive par trois systèmes relationnels : les relations de liens misant sur la communication informelle 
d’affinités (parenté, amitié), les relations de transactions basées sur la communication de proximité et les relations de contrôle axées sur la communication (marketing). Ces stratégies qui représentent de véritables courroies de transmission de l'information d'un point de vue marketing ont fortement contribué à la surpopulation de cette institution académique.

\section{Discussion}

\subsection{Les forces et faiblesses des pratiques de recrutement à l'Université Félix Houphouët Boigny}

- Forces

La question du recrutement des étudiants à l'entrée du système universitaire ivoirien est polémique à double titre. D’un côté, la massification des effectifs étudiants depuis les années 2000 et la contrainte exercée par les performances scolaires sur la réussite professionnelle ont intensifié l'enjeu constitué par les études supérieures. La sélection à l'entrée de l'Université Félix Houphouët-Boigny se fait pour l'essentiel sur la base des performances et critères scolaires. La seule modalité de sélection en vigueur est l'analyse des résultats scolaires des nouveaux bacheliers.

Cette modalité se résume essentiellement à l'analyse du profil des nouveaux bacheliers en fonction des moyennes d'orientation en vigueur et de la capacité d'accueil des différentes unités de formation et de recherche (UFR) et de l'âge du candidat qui est de 23 ans au maximum.

Cette conception méritocratique de l’enseignement supérieur, qui conduit à une sélection des étudiants à partir de critères scolaires dans le contexte d'une université publique donne à chacun les mêmes chances d'accéder à l'enseignement supérieur ivoirien. Elle préfigure l'affrontement de deux conceptions de la justice sociale : le mérite et l'égalité, car l'Université Félix-Houphouët-Boigny est un vaste territoire d'environ 200 ha constitué de treize unités de formation et de recherche (UFR) :

- (Biosciences, Criminologie, Informations, Communication et Arts, Langues, Littératures et Civilisations, Mathématiques et Informatiques ,Odontostomatologie, Sciences de la Terre et des Ressources Minières, Sciences de l'Homme et de la Société, Sciences des Structures de la Matière et de Technologie, Sciences économiques et de Gestion, Sciences Juridiques, Administratives et Politiques, Sciences Médicales et les Sciences Pharmaceutiques et Biologiques) ;

- de deux centres de recherche autonomes (CIRES, CURAT) ;

- et une école de formation continue : le Centre Universitaire de Formation Permanente (CUFOP). 
En clair, l'orientation des étudiants et plus généralement des élèves à l’Université Félix Houphouët-Boigny se réalise en théorie dans une perspective utilitariste.

- Les faiblesses

Face à l'intensification de la concurrence sur le marché de l'enseignement supérieur, l'Université Félix Houphouët-Boigny se retrouve, de fait, confrontée à la nécessité d'améliorer ses stratégies de communication, de promotion, de marketing et de recrutement des étudiants. Ses opérations de recrutement des étudiants présentent des insuffisances. Sa massification a modifié les caractéristiques et la capacité du recrutement des différentes filières. Les politiques de recrutement sont à visée quantitatives et compensatoires. La réussite des étudiants et la gestion des effectifs doivent être planifiées suivant un processus continu d'adaptation et de changement. C'est pourquoi les pratiques de recrutement méritent d'être formalisées et améliorées à l'image des universités américaines (Université de Berkeley) qui procèdent par des tests pour le recrutement des effectifs étudiants : le scholastic aptitude test (SAT) et la modalité des quotas. L'université de Berkeley en Californie fera un usage progressif du SAT en l'utilisant dans un premier temps pour sélectionner les étudiants boursiers, extérieurs à l'État californien, afin de faire entrer son usage dans les mœurs. C'est en 1967 que le SAT fut officiellement adopté comme outil de sélection des entrants en première année.

Quant à la politique des quotas, elle a permis aux universités anglosaxonnes de corriger les inégalités sociales durant le recrutement de la clientèle étudiant.

Il convient de noter que la Gestion stratégique des effectifs affecte directement les ressources des établissements. Le nombre d'étudiants, ainsi que leur réinscription (fidélisation) génèrent des revenus par le biais des droits d'inscription. Au-delà de cet aspect financier, la présence même des étudiants contribue largement à définir la culture des établissements.

\section{Conclusion}

La communication institutionnelle représente un véritable outil de vulgarisation du processus de recrutement des effectifs étudiants à l'université Félix Houphouët-Boigny à travers trois composantes (la communication informelle d'affinité, la communication de proximité et le marketing). La sélection à l'entrée de cette institution se fait à partir d'une vision méritocratique qui se résume pour l'essentiel à l'analyse de dossiers et du profil de la clientèle étudiante en fonction des moyennes d'orientation et de la capacité des infrastructures d'accueil.

Cette stratégie (vision méritocratique) présente cependant des insuffisances, car le recrutement de façon parallèle de milliers d'étudiants 
vient faire déborder largement la population d'étudiants: source de dysfonctionnement et de dégradation de l'image institutionnelle de l’université Félix Houphouët-Boigny.

C'est pourquoi dans ce contexte de mise en concurrence des établissements où les principes de démocratisation de l'université s'effacent derrière les maitres mots de compétitivité, productivité et flexibilité, il serait indiqué que les autorités universitaires de Cocody construisent une bonne politique de recrutement afin de faire bénéficier à l'institution une image positive. C'est dans cette dynamique que Stensaker (2007:13) avance qu'une: " bonne image peut avoir une incidence positive sur les inscriptions des étudiants, le recrutement du personnel enseignant, les ressources et le capital sympathie dont jouit l'établissement ».

En attendant, l'Université Félix Houphouët-Boigny doit peut-être commencer par devenir elle-même un champ expérimental des théories qu'elle enseigne aux étudiants pour mieux organiser son autonomie, sa gouvernance et le recrutement de sa clientèle étudiante.

\section{References:}

AGRAIN René (1949), " Histoire des Universités », Paris, 125 p ; BLÉ, Raoul Germain (2009), «la crise des universités publiques dans la crise ivoirienne : analyse du discours du $1^{\mathrm{er}}$ mai 2007 de Laurent Gbagbo président de la République de Côte d'Ivoire », in Revue Tunisienne de Communication Institut de Presse et des Sciences de l'Information, Tunis, La Manouba ;

Conseil de Gestion de l’Université de Cocody: rapport d'activités de l'ordonnateur pour les années universitaires 2004-2005 ; 2006-2007 ;

ESSANE Séraphin (2001), " une sociologie de l'université en Afrique », Abidjan, 200p ;

FOURASTIÉ Jean (1972), « faillite de l’université ? » Paris, 186p ;

GADAL N (1992), « le mémento de la communication», Paris, Roudil, 90 p ; GOIN Bi Zamblé Théodore (2011), « liberté académiques, syndicalisme et politique en Côte d'Ivoire », JHAE / RÉSA vol $9 \mathrm{~N}^{0} 1 \&$ 2, PP.133-146 ; LAMIZET, Bernard ; SILEM Hamed (1997), « Dictionnaire encyclopédique des sciences de l'information et de la communication », Paris, 590p ;

LAVIGNE Alain (2004), « la communication de recrutement à l'université Laval : une lecture relationnelle des stratégies implicites et explicites ainsi que quelques a priori au recours au (public interne) étudiant, communication et organisation no 24 ;

LAVIGNE Alain(2005), « le recrutement des effectifs étudiants à l'université Laval: une analyse du déploiement des stratégies de la communication institutionnelle " Communication [En ligne], Vol. 23/2 |, consulté le 25 octobre 2005 ; 
LIBAERT Thierry (2009), "Introduction à la Communication », Paris, 122p ;

MILON, Alain ; JOUVE Michèle (1996), " Communication et organisation des entreprises : approches critiques et cas pratiques ", Edition Bréal, paris, 287p ;

N’DA, Paul (2006), « Méthodologie de la recherche, de la problématique à la discussion des résultats », Abidjan, 159p ;

STENSAKER Bjorn, les liens entre l'image de marque et l'évolution des organisations, politiques et Gestion de l'Enseignement Supérieur, $2007 \mathrm{~N}^{\mathrm{O}}$ 19 p 13-30 ;

TEDGA, Paul John Marc (1988), «l'enseignement supérieur en Afrique noire, la catastrophe », Paris,223p ;

VALY Diarrassouba Charles (1979), «l'université ivoirienne et le développement de la nation ", Abidjan, $214 \mathrm{p}$;

ZINSOU Edme Michel (2009), «l'université de Côte d'Ivoire et la société », paris, 318p ;

\section{Notes}

1-information tirée dans l'article de Jean-Alain Goudiaby : le Sénégal dans son appropriation de la reforme LMD.

2-PAES / UEMOA/ enquêtes auprès des établissements d'enseignement supérieur, juin 2011 et novembre-décembre 2011

3-statistiques des inscriptions de l'année académique 2008-2009 de la scolarité centrale de L'université Félix Houphouët-Boigny

4- effectifs des enseignants-chercheurs permanents et contractuels de la direction des Ressources humaines de l'université Félix Houphouët-Boigny 5-_statistiques des inscriptions de l'année académique 2008-2009 de la scolarité centrale de L’université Félix Houphouët-Boigny

6-Conseil de Gestion de l'Université de Cocody : rapport d'activités de l'ordonnateur pour les années universitaires 2004-2005 ; 2006-2007 7- Conseil de Gestion de l'Université de Cocody : rapport d'activités de l'ordonnateur pour les années universitaires 2004-2005 ; 2006-2007 